\title{
ON SOME SPECTROGRAPHIC OBSERVATIONS RELATED TO THE STRUCTURE WITH HEIGHT OF ACTIVE REGIONS AND PARTICULARLY SOLAR FLARES
}

\author{
YNGVE ÖHMAN \\ (Stockholm Observatory, Saltsjøbaden, Sweden)
}

\begin{abstract}
A BSTRACT
A peculiar kind of double-peaked $\mathrm{H} \alpha$ lines in solar flare spectra is described. The most likely explanation to this phenomenon is an absorption in the centre of the line due to mottles and fibrils situated above the flare. Another explanation might be a rapid rotatory mass motion in the filamentary structure of the flare itself. In some other flare spectra a slight inclination of the $\mathrm{H} \alpha$-emission streak has been found indicating as well that rotatory mass motions may be present in flares.
\end{abstract}

The writer has described before (1) a peculiar $\mathrm{H} \alpha$ spectrum of a flare of importance $1 \mathrm{~b}$ observed in Anacapri on March 29, 1966 (UT 09.52) showing a double-peaked H $\alpha$ line, similar to that observed in the low chromosphere at the limb. This interesting spectrum is presented again in Figure la. The emission streak is seen in the centre of the $\mathrm{H} \alpha$ line also but here the streak is not only weaker but also clearly narrower than in the two well-defined emission peaks. Therefore these peaks, though situated in the steep slope of the $\mathrm{H} \alpha$ photospheric line, can hardly be due to an apparent intensity increase produced by the increase in background intensity, but must be due to a real emission feature of the flare spectrum. The distance between the peaks is about $1 \cdot 2 \AA$. The flare was situated very near a sunspot with the coordinates $26 \mathrm{~N}, 59 \mathrm{E}$.

In the same photographic recording of the solar spectrum a flare spectrum with moustaches (2) is seen. It is situated slightly above the same spot.

We have found several examples of the double-peaked $\mathrm{H} \alpha$ line-flare spectra. In Figure $1 \mathrm{~b}$ a similar, though fainter object is seen, which was obtained at the Swedish Solar Observatory in Anacapri on July 8, 1966 at UT 12.56. The distance between the peaks is about the same as in Figure la, but the peaks are not quite so narrow resembling more or less a necktie.

Sometimes objects of this kind are connected with dark surges and may in fact be bright surges visible on the disk. Figure $2 b$ obtained on April 5, 1967 at UT 10.07 would suggest a bright surge phenomenon combined with dark surges. But the emission features had a much longer duration than the dark ones, which is evident from Figure 2 a obtained at UT $09 \cdot 17$ and Figure $2 \mathrm{c}$ at UT 10.23 and some later exposures. So at least the object in Figure 2 seems to have been more related to a flare than to a bright surge. In fact, it coincided with a small brilliant plage in $25^{\circ} \mathrm{S}, 40^{\circ} \mathrm{E}$ indicating 
(a)

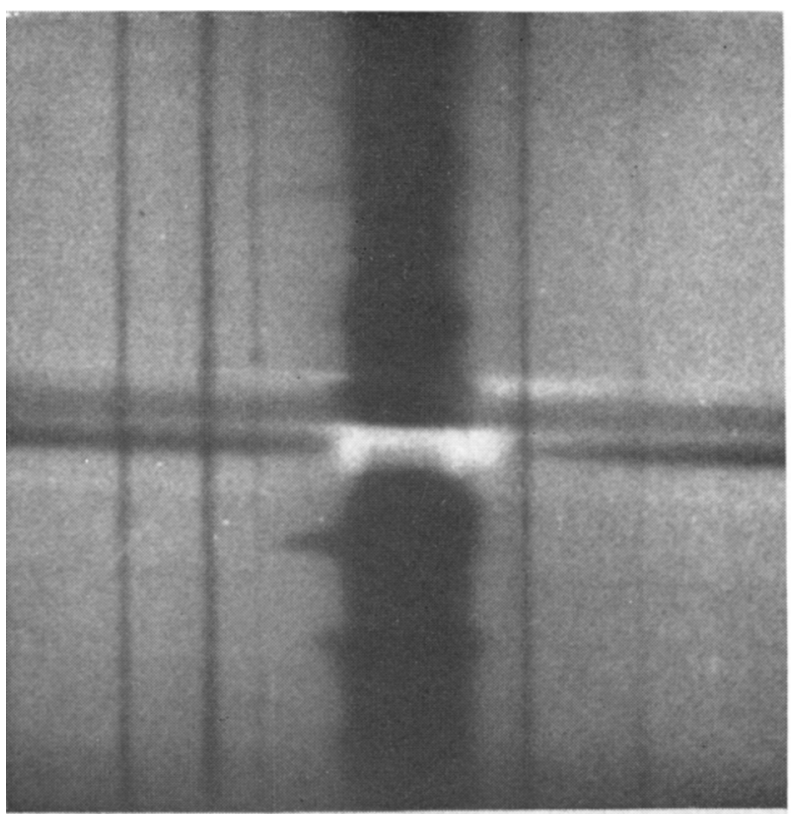

(b)

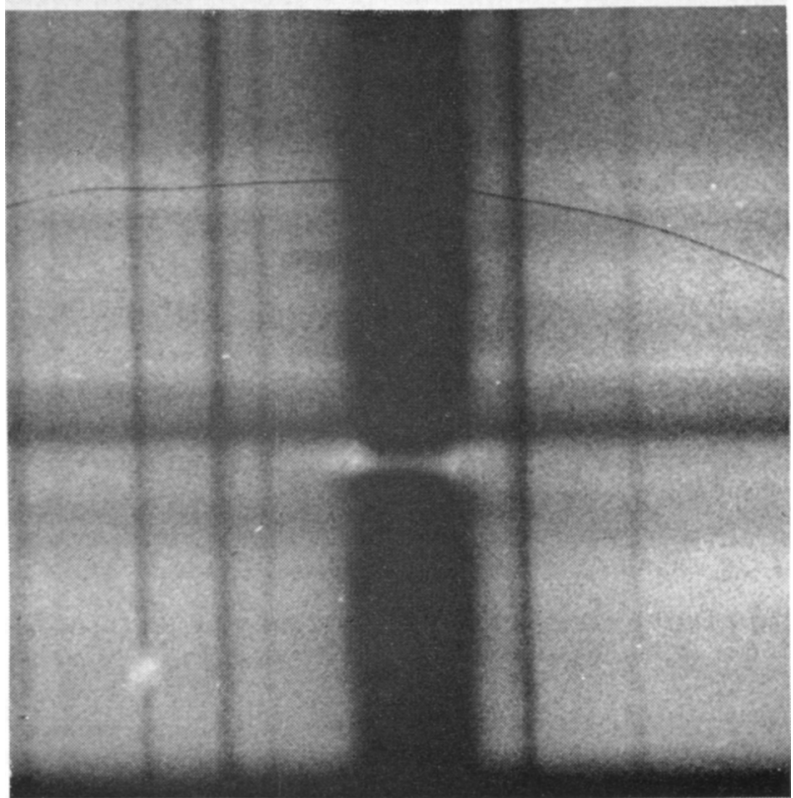

FIG. 1. Double-peaked H $\alpha$ lines in solar flares observed on the disk. la was obtained with a Babcock grating spectrograph on March 29, 1966, whereas 16 was obtained on July 8, 1966. Note also the moustaches in (a) above the flare. 
(a)

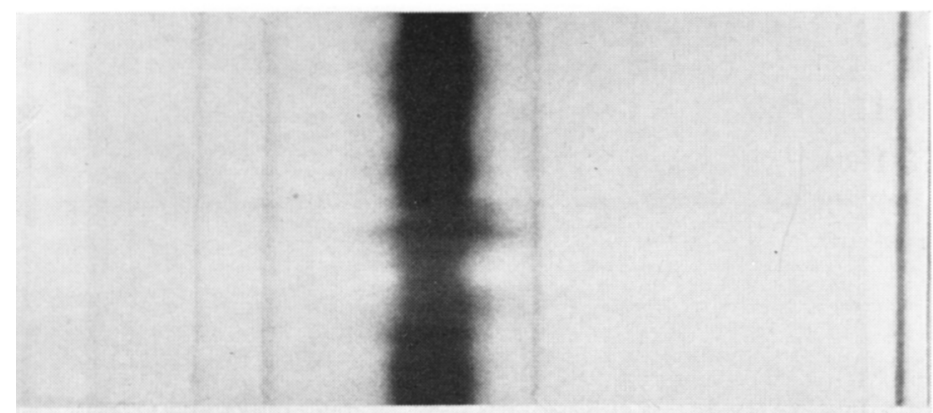

(b)

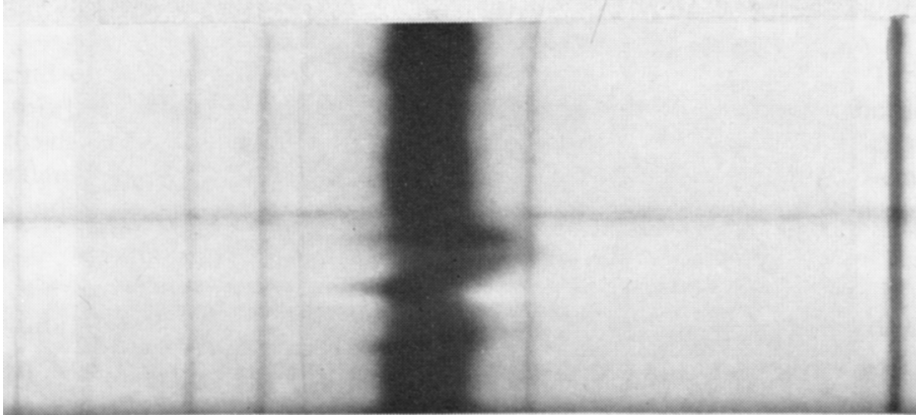

(c)

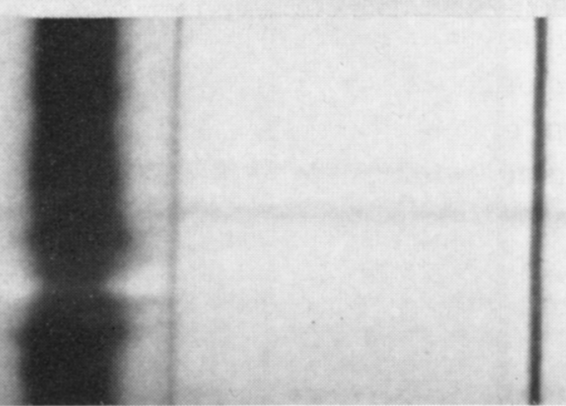

FIG. 2. Spectrum of a brilliant plage accompanying the appearance on April 5, 1967 of a new spot. $2 a$ was obtained at UT 09.17, $2 b$ at UT 10.07 and $2 c$ at UT 10.23. Note the dark surge appearing in $(b)$.

the creation of a new spot. In a way it seems related to the moustaches too, but, if so, the moustaches are rather short.

In an earlier publication (1) I have suggested that the double-peaked flare in Figure la is a low object with some absorbing mottles and fibrils above producing a strong reversal of the emission line in the centre of the line. This kind of central reversal has been suggested by Giovanelli, Michard and Mouradian (3) to be present in the $\mathrm{H} \alpha$ spectrum of the low chromosphere. In fact dark features of even large size can sometimes be clearly traced in the chromospheric spectrum of the limb (1). 
Though the spectra presented in Figure 1 and Figure 2 seem fairly well explained by assuming the objects to be low ones with absorbing mottles and fibrils above, we should not overlook the possibility of mass motions in different directions within the object imaged on the slit of the spectrograph. The possibility of such mass motions in solar flares has been considered before to explain the asymmetry of the $\mathrm{H} \alpha$ emission line, and I want to draw attention particularly to a recent paper by Ballario (4). Mrs. Ballario has considered in this connection flares with loop structure.

One difficulty in explaining the double peak in the spectrum Figure la as a result of rapidly ascending and descending mass motions is the narrow-line profile of each constituent needed to obtain the well-defined peaks. The emission-line features of Figure $1 \mathrm{~b}$ and Figure 2 are perhaps somewhat easier to explain as a result of mass motions only. Indeed, it is tempting to assume a rotatory mass motion with the axis of rotation more or less parallel to the slit of the spectrograph.

We have found recently some independent evidence of rotatory mass motions in flares, and, in fact, because of a slightly inclined $\mathrm{H} \alpha$ streak. Figure $3 \mathrm{a}$ and $3 \mathrm{~b}$ show examples of this phenomenon appearing in an importance $1 \mathrm{~b}$ flare observed by me in Anacapri on July $3,1967\left(20^{\circ} \mathrm{S}, 16^{\circ} \mathrm{E}\right)$. 3a was obtained at UT $07 \cdot 48,3 \mathrm{~b}$ at UT $08 \cdot 09$, $3 \mathrm{c}$, finally, shows an $\mathrm{H} \alpha$-patrol image obtained by $\mathrm{O}$. Gimse at UT 08.20 and with the approximate position of the slit. In addition to a faint inclination due to a remaining error of adjustment of the grating (changes had just been made) the emission streaks show a clear effect of intrinsic inclination. Similar effects have been observed and described by Severny (5). In my opinion it is difficult to explain the inclination of the streak without assuming a mass motion, and a rotatory motion is perhaps well possible. If so, the axis of rotation would be more or less perpendicular to the slit. It is interesting to note in Figure $2 b$ that the inclination is stronger here than in Figure $2 a$. This may be a result of the reduced intrinsic line width, eventually combined with an increased separation of mass elements.

Inclined emission streaks are well known in the spectra of spicules, but have in general been attributed to effects of unresolved superimposed objects with different mass motions (6). But the possibility of a rotatory motion should perhaps be considered in the case of spicules too. Moreover, we have found some $\mathrm{H} \alpha$ spectra of prominences indicating rotatory mass motion, the spectra showing some features in common with Figure 1. That is to say, double emission peaks appear. Figure 4 shows such an object near the limb secured by us in Anacapri on July 2, 1967 at UT 13.31. In fact one of the circular $\mathrm{H} \alpha$ recordings shows a strong 'limb-brightening' not only to the left and right but also above. Self-reversal alone can hardly explain this structure. It is tempting therefore to consider mass motions as well and perhaps of a rotatory type. A study of a great quantity of material may give us a better clue to the understanding of these curious objects and their relation to flares and loopes. If possible, observations should be made simultaneously in different spectrum lines.

The possibility of rotatory mass motions should be considered also from a theoreti- 
(a)

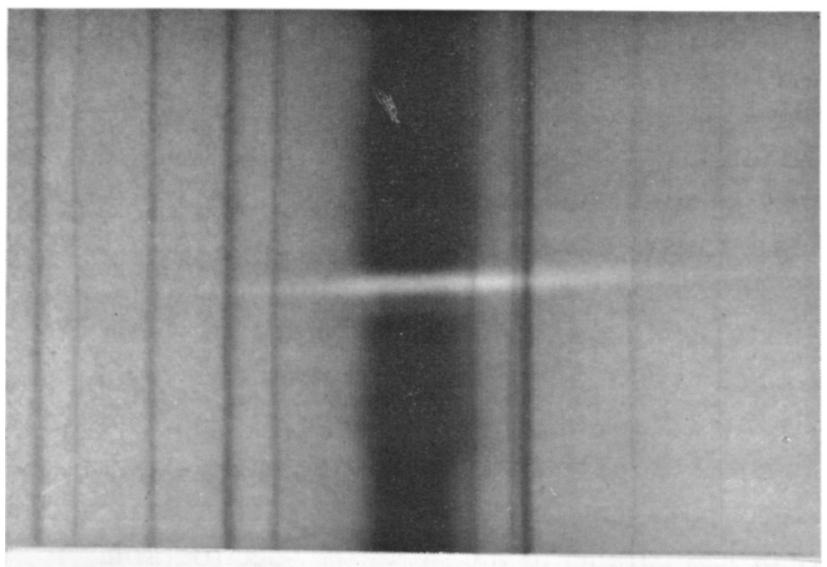

(b)

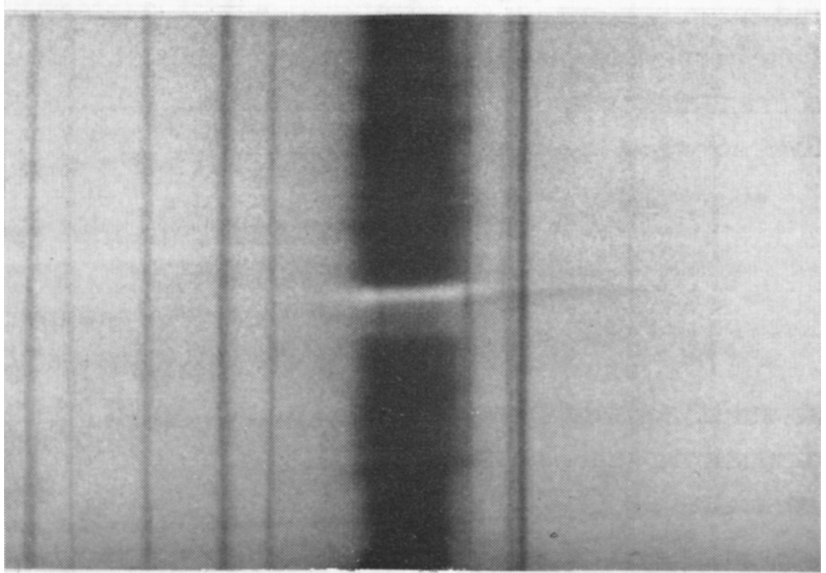

(c)

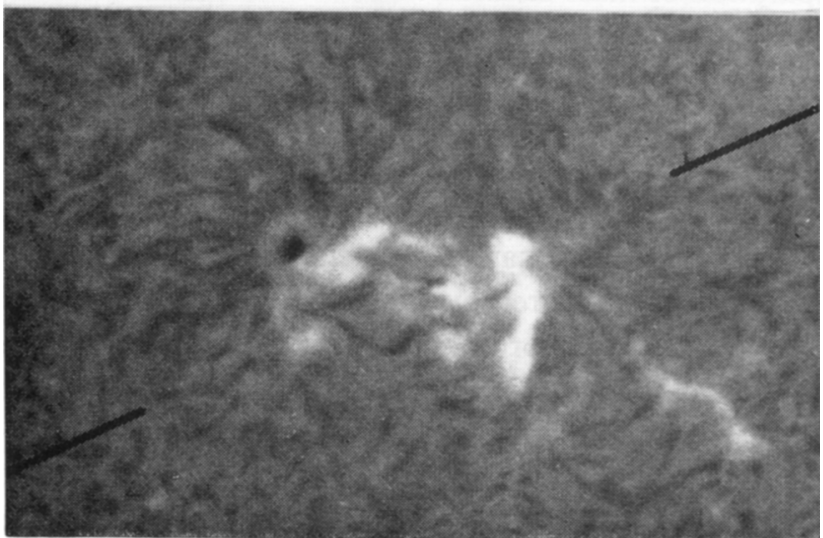

FIG. 3. Spectra of an importance $1 \mathrm{~b}$ flare observed on July 3,1967 at $20^{\circ} \mathrm{S}, 16^{\circ} \mathrm{E}$. 3 a was obtained at UT 07.48, $3 \mathrm{~b}$ at 08.09. $3 \mathrm{c}$ is an Ha picture of the flare at UT 08.20. The inclination of the Ha streaks in $(a)$ and $(b)$ is partly real and indicates mass motion, perhaps by rotation. 
cal point of view. In fact Danielson (7) has considered already roll convection to be present in the fibrous structure of the penumbra in sunspots. Professor Alfvén has kindly informed me that rotatory motions may well be expected in flares according to his and Carlqvist's recent theory of flares (8).

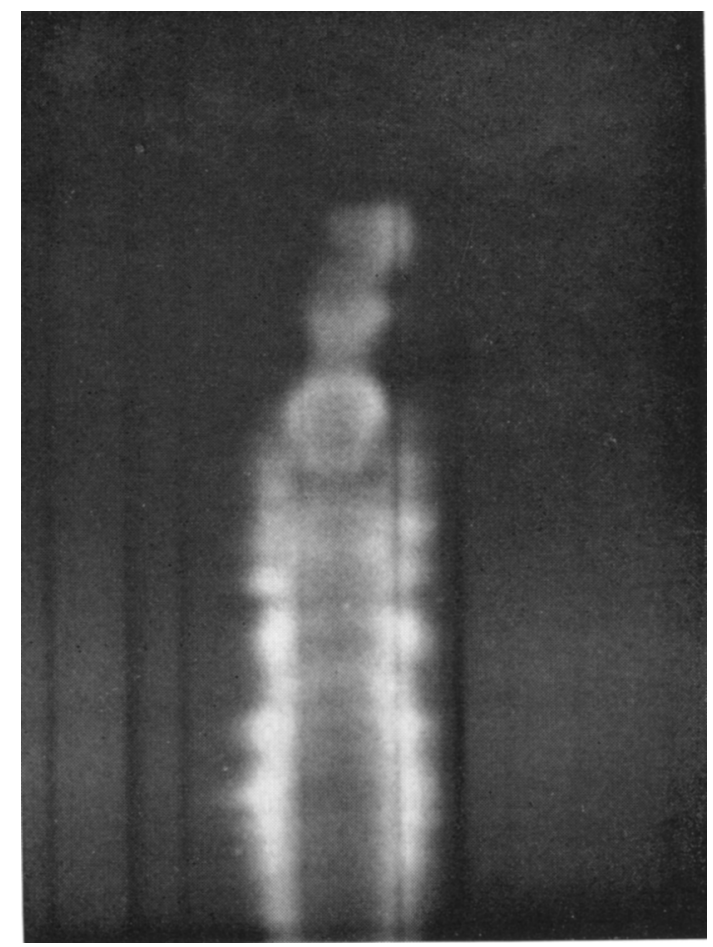

FIG. 4. Ha spectrum of prominences and the chromosphere at the limb obtained on July 2, 1967. Note the circular apparent shape of one of the prominences with a limb-brightening possibly due to mass motion of a rotatory type.

\section{References}

1. Öhman, Y. (1966) Stockholms Observatorium, Meddelande No. 161.

2. Severny, A. B. (1957) Izv. krym. astrofiz, Obs. 17, 129.

3. Giovanelli, R.G., Michard, R., Mouradian, Z. (1965) Ann. Astrophys., 28, 871.

4. Ballario, Maria Cristina (1963) Osservatorio Arcetri, Contr. 78.

5. Handbuch der Physik, Springer, Band LII, Astrophysik, III, p. 206, Fig. 61d, 1959.

6. Beckers, J. M. (1964) Dissertation, Utrecht, p. 72.

7. Danielson, R.E. (1962) Astrophys. J., 134, 289.

8. Alfvén, H., Carlqvist, P. (1967) Solar Phys., 1, 229.

\section{DISCUSSION}

Pasachoff: I agree that many inclined spectral features are visible in limb observations. On spectra taken jointly with Drs. Beckers and Noyes at the Sacramento Peak Observatory and reduced at the 
Harvard College Observatory, such inclined features are visible not only in $\mathrm{H} \alpha$ but also in the $\mathrm{H}$ and K lines of Ca II (J. M. Beckers, R. W. Noyes, and J. M. Pasachoff, Astron. J., 71, 1966, 255).

Our spectra were made with a dispersion of $0.15 \AA / \mathrm{mm}$ and a $25-\mathrm{cm}$ solar image with the 16-inch coronagraph and 13-m Littrow spectrograph and sequences exist with exceptional seeing. We believe that we can satisfactorily resolve individual spicules and that many of the inclinations are real. Sometimes inclined features are visible at great heights, where the total number of features is small and the chances of accidental overlapping minimized.

We interpret these inclinations as arising from differential mass motions, perhaps from rotation around the spicular axes.

The inclinations at some features change with time. This can be interpreted as a real change in the velocity, a change in the orientation of the feature with respect to the line of sight or a change in the width of the line profile.

Further, our $\mathrm{H}$ and $\mathrm{K}$ spectra include a disturbed region in the chromosphere we used an image slicer to give us effectively two slits located approximately 4000 and $8000 \mathrm{~km}$ above the limb. This allows us to follow the relation of the simultaneous line-of-sight velocity at the two levels.

Exposures were $13 \mathrm{sec}$ long for $\mathrm{H}$ and $\mathrm{K}$ and were taken every $30 \mathrm{sec}$. The disturbed region extended about $40000 \mathrm{~km}$ along the slit.

For several minutes, the upper level is seen to contain a disturbed area, for there are many knots and short, highly inclined features indicating the presence of strong differential motions. The lower level has normal spicular features. Eventually it too begins to show abnormal structures.

Then, on the next frame, a feature becomes visible at the lower level with a line-of-sight velocity of about $50 \mathrm{~km} / \mathrm{sec}$. On this frame, the feature is very faint in the upper level and has a somewhat lower velocity, about $35 \mathrm{~km} / \mathrm{sec}$.

On the following frame, $30 \mathrm{sec}$ later, the feature has become very bright in the upper level, with a large Doppler velocity of $60 \mathrm{~km} / \mathrm{sec}$. The velocity in the lower level has declined to about $15 \mathrm{~km} / \mathrm{sec}$, although the profile remains wide.

On the next frame, the velocity in the upper level increases slightly to about $70 \mathrm{~km} / \mathrm{sec}$, while the velocity in the lower level declines to about zero. This is, unfortunately, the end of the time sequence.

Note that the velocity of propagation implied is about $4000 \mathrm{~km}$ in $30 \mathrm{sec}$ or $130 \mathrm{~km} / \mathrm{sec}$. This is far more than supersonic, but is comparable to the Alfvén speed for reasonable chromospheric densities and magnetic fields. Other events visible at both lower and upper levels do not usually show even a time difference of this magnitude, indicating still higher velocities.

Maltby: At the Oslo Solar Observatory we have observed inclined elements in $\mathrm{H} \alpha$ in the quiet chromosphere, close to sunspots as well as in flares. Several explanations, in addition to rotatory motion, are possible.

Bappu: I would just like to make a comment on the inclined structures seen in $\mathrm{H} \alpha$. When one obtains $\mathrm{H} \alpha$ spectra of the centre of the solar disk with good image and spectrographic resolution, one can see many narrow inclined structures in absorption. Rotation of the mass element is an obvious interpretation, and one can postulate the mass to be either experiencing a helical motion or be in a form where one has ascending and descending columns side by side.

Ohman: I am glad to hear that several observers have found similar effects, and I am fully aware of the fact that different interpretations are sometimes possible. 